\title{
Dispersion and rheological studies of Y-PSZ tape casting slurry
}

\author{
K G VASANTHA KUMARI, K SASIDHARAN, M SAPNA and RAGHU NATARAJAN* \\ Centre for Materials for Electronics Technology (C-MET), Athani PO, Thrissur 680 771, India
}

MS received 6 August 2004; revised 7 January 2005

\begin{abstract}
Different solvent systems in combination with three different dispersants were tried to find out the suitable solvent-dispersant combination, which give optimum dispersion of PSZ. Based on sedimentation, viscosity and rheology characteristics, zeotropic ethanol : xylene with a ratio of $50: 50$ along with 0.5 wt $\%$ phosphate ester was found to be the best solvent and dispersant combination. Optimized tape casting slurry was prepared using PEG 600 and BBP as plasticizers and PVB as the binder. Cyclohexanone was used as the homogenizer. The optimized slurry composition with $\mathbf{5 8 \%}$ solid loading exhibited shear-thinning pseudoplastic rheological behaviour. Y-PSZ tapes of $\sim 50 \mu \mathrm{m}$ thickness free from visible defects were cast with a green tape density of $55 \%$.
\end{abstract}

Keywords. Tape casting; dispersability; sedimentation; rheology.

\section{Introduction}

Zirconia is known as an 'all rounder' advanced ceramic material (Heuer and Hobbs 1981; Somia et al 1988; Deb and Radhakrishna Bhat 2000; Kernan et al 2003). This is because the material can be tailored to assume very different properties for a wide range of advanced ceramic products. This stem from the interesting variety of properties that can be achieved, with different alloy compositions and heat treatments. Much attention has been devoted to the development of $\mathrm{ZrO}_{2}$ based ceramics in the system $\mathrm{Y}_{2} \mathrm{O}_{3}-\mathrm{ZrO}_{2}$. $\mathrm{Y}_{2} \mathrm{O}_{3}$ partially stabilized $\mathrm{ZrO}_{2}, \mathrm{Y}-\mathrm{PSZ}$ contains a mixture of cubic (C) and tetragonal $(t)$ or monoclinic $(m)$ phases, and find use as thermal barrier coatings, because of their low thermal conductivity, or as a solid electrolyte because of their good ionic conductivity (Kernan et al 2003). Zirconia based materials can be exploited for many applications either in their bulk form or through the fabrication of thin layers. Electronic applications, especially solid oxide fuel cells, oxygen sensors and structural composites (e.g. $\mathrm{Al}_{2} \mathrm{O}_{3} / \mathrm{ZrO}_{2}$ ), demand $\mathrm{Y}-\mathrm{PSZ}$ in the form of tapes (Deb and Radhakrishna Bhat 2000). Thin green tapes of the order of 50-150 micrometer thick, is the first step in realization of these devices.

The doctor blade tape casting process (Mistler 1998; Moreno 1992a,b; Andreola et al 2002) is a low cost process for the manufacture of large areas of thin ceramic sheets of controlled thickness and high quality. Tape casting of different materials requires different slurry formulations, which include solvents, dispersants, binders, plasticizers and homogenizers to produce high quality products. To optimize the formulation, a better understanding

*Author for correspondence (rkg124@ rediffmail.com) of the interaction between ceramic powders, organic additives and solvent as well as a better understanding of the influence of their interaction on the processing step is desired. Both non-aqueous and aqueous based routes can be attempted for tape casting of ceramics. Organic-solvent based tape casting systems are widely used mainly because one can obtain improved quality of tape and because of easy and fast evaporation of solvents (Moreno 1992a).

The most important characteristics of a tape casting slurry are (i) a well dispersed homogenous stable system, (ii) minimum viscosity, (iii) shear thinning behaviour and (iv) high solid loading (Feng and Dogan 2000; Bhaskar Reddy et al 2002). The degree of dispersion, deagglomeration and dispersion of the powder in the solvent have profound effect on the microstructure of the green body. Breaking of agglomerates could be achieved by mechanical agitation of the powder. Dispersion of fine ceramic powder in a liquid medium is usually achieved by the addition of optimum amount of dispersant (Bindu and Raghu 2004). For maximum effectiveness the dispersant must have access to each particle surface. The repulsive interaction can be provided by two different general mechanisms or a combination thereof. One is electrostatic repulsion as a result of development of an electrical double layer around each particle upon dispersing a powder into a polar liquid. This produces a repulsive force, which decreases with increasing separation between particles. The second one is polymeric stabilization in which the stability is conferred by long chain polymers adsorbed onto the particle surface. The dispersion and stability of the suspension are achieved when the repulsive forces are high enough to dominate over the attractive London-van der Waals forces.

Sedimentation technique, a well accepted method to establish the degree of particle dispersion and packing, 
gives a visual representation of deflocculation and dispersion (Chartier et al 1993; Bindu and Raghu 2004). The efficiency of dispersion is evaluated by slower settling rate and higher packing density.

During the dispersion stage, as far as the rheological characterization is concerned, the system that gives the minimum in viscosity and near-Newtonian flow behaviour is considered as the best dispersed condition (Annika Kristoffersson 1999; Olieveria 2001; Jiyou et al 2002). An important characteristic of tape casting slurry is its rheological characteristics, i.e. flow behaviour. Optimized final tape casting slurry composition exhibits pseudoplastic rheological characteristics.

The present work aims at arriving at optimum dispersion condition for Y-PSZ in different combinations of solvents and dispersants. Based on the optimized dispersed condition, tape-casting slurry with shear-thinning rheological characteristics were achieved. Using the optimized tape casting slurry with $58 \%$ solid loading, tapes free from visible defects of $\sim 50 \mu \mathrm{m}$ thickness with a green density of $55 \%$ was obtained.

\section{Experimental}

\subsection{Starting material}

The zirconia powder used for the present study was yttriastabilized zirconia (supplied by ACC, Mumbai, India). The average particle size, as given by the manufacturer, was $\sim 1$ micrometer. In the case of oxides, the most common solvent combination used for tape casting was azeotropic mixtures of solvents with alcohol as one of the constituents (Moreno 1992a). The solvent systems chosen for the present study were mixtures of ethanol $(\mathrm{EtOH}$, Hayman, UK) or isopropanol (IPA, Merck, India) with toluene (tol, BDH, India), methyl ethyl ketone (MEK, Nice, India), or xylene (xyl, BDH, India). The most effective dispersants for oxides that were commonly used in tape casting such as Menhaden fish oil (MFO, Tape casting warehouse), Phosphate ester (PE, Tape casting warehouse) and Triton-X-100 (T-X-100, Octylphenoxypolyethoxyethanol, Merck) were chosen for the present study. For the preparation of tape casting slurry, polyethylene glycol 600 (PEG, Merck, India) and benzyl butyl phthalate (BBP, Sigma Aldrich, India) were used as plasticizers. $20 \mathrm{wt} \%$ polyvinyl butyral (PVB, Sigma Aldrich, MW 60,000 to 1,00,000) was used as binder.

\subsection{Slurry preparation}

Dispersion studies were carried out with $33 \mathrm{wt} \%$ of solid content. With the varied wt $\%$ of dispersant, the slurry was ultrasonicated for $10 \mathrm{~min}$, then ball milled for $24 \mathrm{~h}$ with zirconia balls as milling media for effective deagglo- meration and dispersion. Sedimentation and rheological studies were then carried out using the dispersed slurry containing $33 \mathrm{wt} \%$ solid content.

\subsection{Sedimentation studies}

For sedimentation study, $10 \mathrm{ml}$ of the ultrasonicated and ball milled slurry was immediately transferred to a graduated measuring cylinder and then the slurry was allowed to settle undisturbed for weeks. The sediment height $(H)$ was recorded at regular intervals as a function of time against the initial height $(H 0)$. The readings were noted every $5 \mathrm{~min}$ for the first one hour and then every $15 \mathrm{~min}$ for the next $4 \mathrm{~h}$. Thereafter, $H$ values were noted every hour till $24 \mathrm{~h}$. The final packed-bed height was noted after two weeks.

\subsection{Rheological studies}

Rheological characterization was carried out using a shear controlled Rheometer (Brookefield Programmable DV III + Rheometer) using UL adapter for dispersion studies and small sample adaptor (Spindle SC4-18) for tape casting slurry. The general flow behaviour was studied by plotting the variation of viscosity with shear rates. These viscosity data were tested by fitting with different mathematical models using the Rheocalc Software (Brookefield Engineering, USA). Casson's equation gave the best fit (Annika Kristoffersson 1999; Paik et al 1999). From this, the value of yield stress can be readily calculated. This value helps us to quantitatively compare the flow behaviour of different samples. Lower viscosities and lower yield stress values indicate a stable system. In the case of final tape casting slurry shear-thinning behaviour is desirable as they experience shear force under the action of a moving blade/carrier.

\subsection{Tape casting}

Tape casting slurry was prepared with the optimum concentration of the selected solvents and dispersant. Doublestep slurry preparation was carried out. At the first stage, powder, solvent and dispersant alone were ball milled with zirconia balls as milling media for $4 \mathrm{~h}$. This treatment was effective in breaking up the soft agglomerates and to ensure equilibrium adsorption of dispersant on the powder surface. Other tape casting additives were then added and the slurry was rolled for $24 \mathrm{~h}$.

Tape casting was carried out using double doctor blade technique on a clean glass bed using a laboratory batch type tape caster (EPH Engineering, USA). The blade gap was kept at $250 \mu \mathrm{m}$. After natural drying, tapes were released and inspected for potential defects. Thickness and green density were measured. 


\section{Results and discussion}

\subsection{Selection of solvent mixture}

It is well recognized that apart from the nature of dispersant, proper combination of solvents with desired characteristics, especially its polarity play a vital role in dispersing ceramics (Badini et al 2001; Bhaskar Reddy et al 2002). Hence to understand the effect of solvent alone, initially different combinations of solvents were tried for dispersing PSZ without the addition of dispersant. Figure 1 shows the sedimentation results of PSZ with IPA as one of the solvent systems. For better clarity the initial rate of sedimentation for the first one hour and the final $H / H O$ after two weeks are shown. It is clearly observed from figure 1 that IPA-tol system exhibits slower rate of sedimentation compared to the other systems. The final $H / H O$ was also found to be the lowest for this system. Figure 2 represents the initial sedimentation curves of EtOH based solvent systems. In this case different ratios of EtOH to xylene was tried as they belong to zeotropic system. As can be seen EtOH-xyl of $50: 50$ ratio exhibited the slowest rate of sedimentation and lowest final $H / H 0$. Hence for further studies, IPA-tol and EtOH-xyl (50:50) were chosen.

To understand the effect of different dispersants, initially sedimentation and viscosity studies were carried out with $1 \mathrm{wt} \%$ of the dispersant. In the case of IPA-tol system while for T-X-100 and MFO the slurries settled immediately, for PE system it took a few days for settling (figure 3). The final $\mathrm{H} / \mathrm{HO}$ is also the lowest for PE system. In the case of EtOH-xyl system also, PE showed the lowest settling rate (weeks for settling) and lowest $H / H 0$ (figure 4).

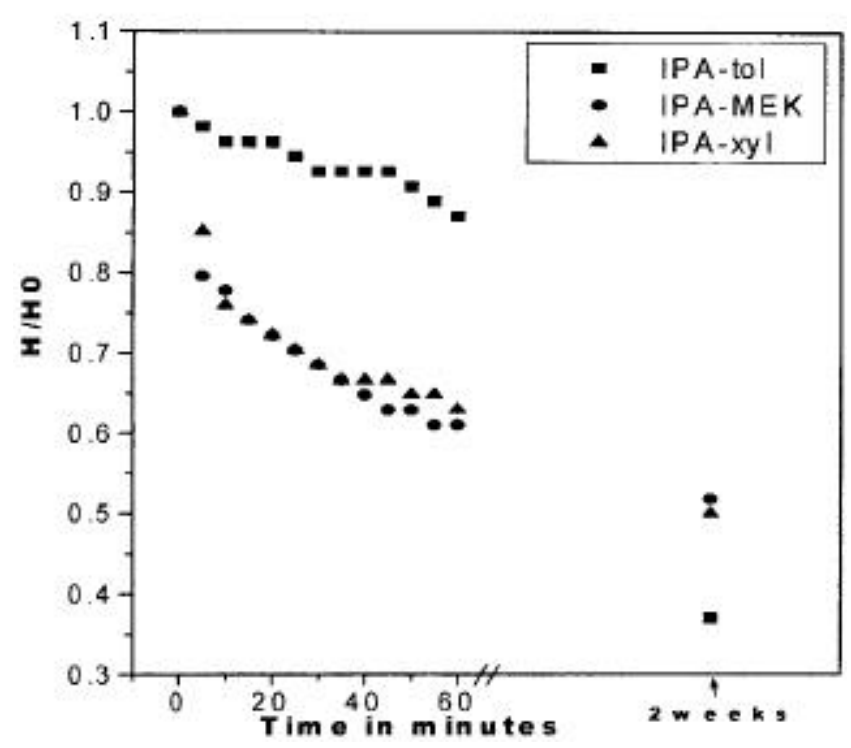

Figure 1. Sedimentation study of $\mathrm{ZrO}_{2}$ with IPA based solvent systems.
As can be seen from figure 5 for the IPA-tol based system, PE exhibited the lowest viscosity as well as nearNewtonian rheological characteristics. Figure 5 also supports the fact that in the case of EtOH-xyl system also PE is the best dispersant. Comparing IPA-tol-PE with EtOH-xyl$\mathrm{PE}$, the latter system exhibits the lowest viscosity and near-Newtonian behaviour. The yield stress values are also the lowest for this system (table 1). Hence for further studies the latter system is selected.

To understand the effect of concentration of PE on the dispersability of the powder, sedimentation (figure 6) and viscosity (figure 7) studies were carried out with varying

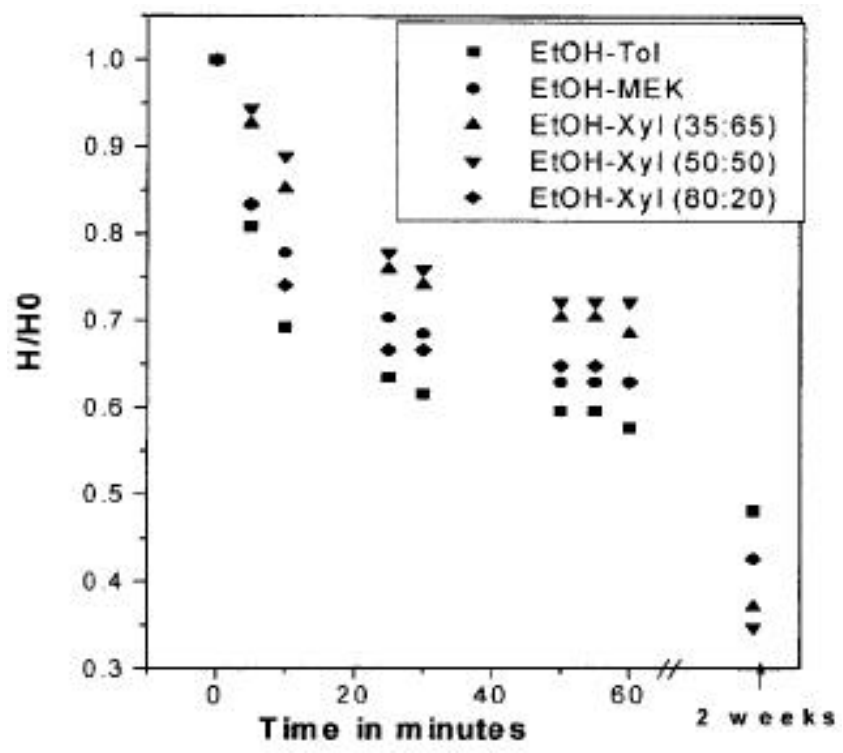

Figure 2. Sedimentation study of $\mathrm{ZrO}_{2}$ with EtOH based solvent systems.

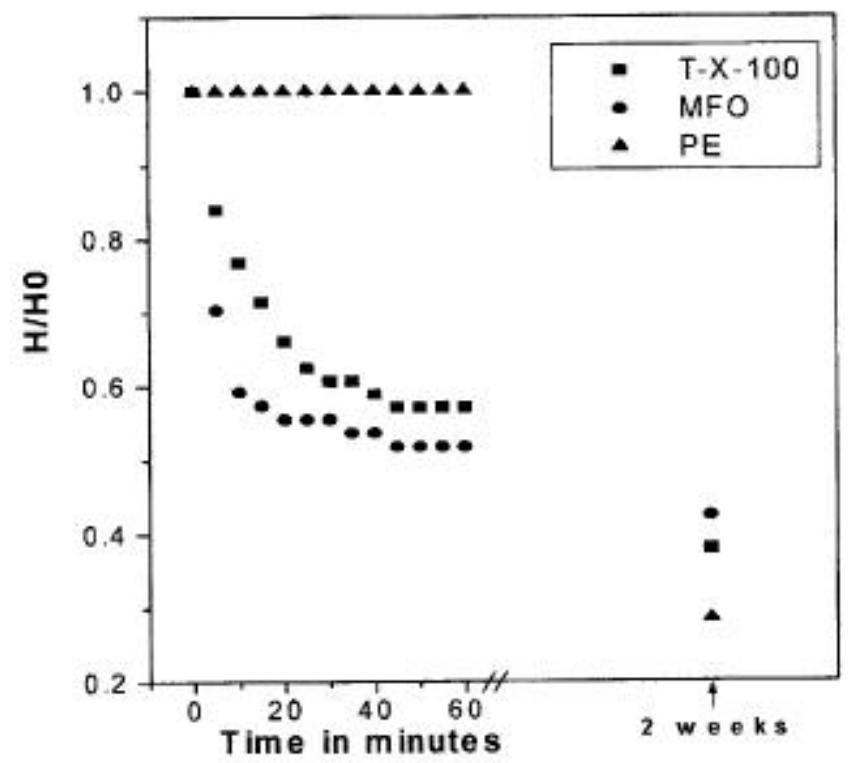

Figure 3. Sedimentation curves for $\mathrm{ZrO}_{2}$ in IPA-tol system with different dispersants. 
$\%$ of PE from 0 to $2 \mathrm{wt} \%$. With increasing concentration of $\mathrm{PE}$ up to $0.5 \mathrm{wt} \%$ the rate of sedimentation became the lowest and the final $H / H O$ also showed the lowest i.e. maximum final packing density is observed for $0.5 \mathrm{wt} \%$. Yield stress values (table 2) also confirm that $0.5 \mathrm{wt} \%$ is the optimum dispersant concentration. With further increase in $\mathrm{PE}$ concentration, the rate of sedimentation increased and the final $\mathrm{H} / \mathrm{HO}$ was also higher. Final $\mathrm{H} / \mathrm{HO}$ started increasing. Similar trend is clearly seen in viscosity behaviour also. Upto $0.5 \mathrm{wt} \%$ the system showed lower viscosities and beyond $0.5 \mathrm{wt} \%$ the viscosity increased. It is also observed that $0.5 \mathrm{wt} \%$ PE system shows perfect Newtonian behaviour, which is highly desirable at the dispersion stage. The optimum amount of dispersant cor-

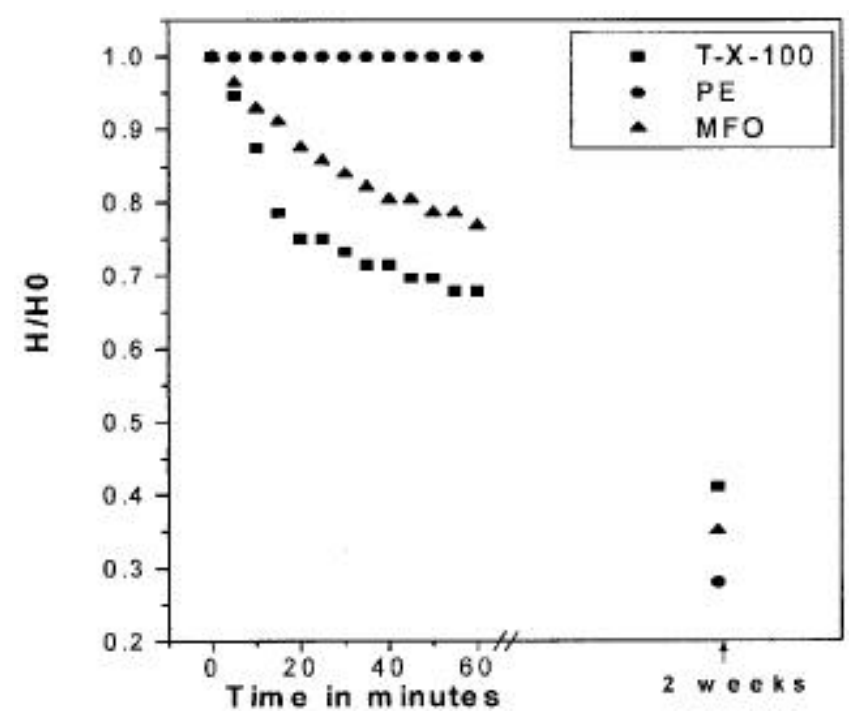

Figure 4. Sedimentation curves for $\mathrm{ZrO}_{2}$ in $\mathrm{EtOH}-\mathrm{xyl}$ system with different dispersants.

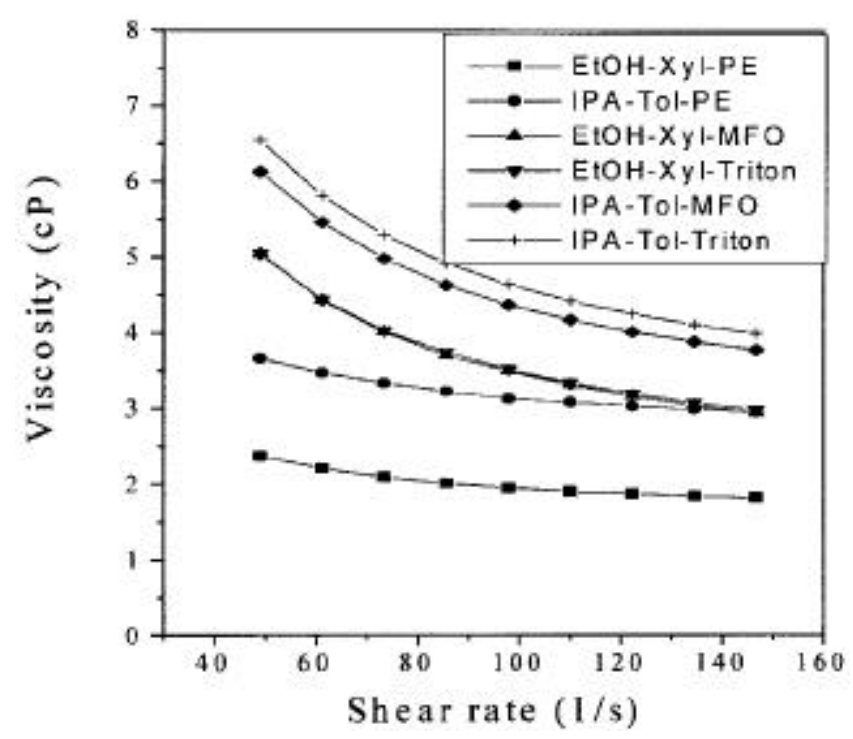

Figure 5. Viscosity of $\mathrm{ZrO}_{2}$ with different dispersants. responds to the amount giving monolayer coverage on the particles. A further increase of the dispersant concentration corresponds to a condition where saturation adsorption limit exceeds and the presence of excess polymer leads to particle destabilization by a bridging mechanism (Zhu et al 2003).

Tape casting slurry composition was optimized using PEG and BBP as plasticizers, PVB as binder, using EtOH$\mathrm{xyl}$ as the solvent and PE as the dispersant. Cyclohexanone was used as the homogenizer. The optimized slurry composition is shown in table 3. Figures 8 and 9 show the rheological characteristics of tape casting slurry at every stage of addition and that of the final tape casting slurry having 58\% solid loading. As expected the final tape casting slurry exhibited shear-thinning pseudoplastic behaviour. Using the optimized slurry composition, green tapes free from visible defects of $\sim 50 \mu \mathrm{m}$ thickness were obtained (figure 10) with a green density of $55 \%$.

Table 1. Effect of different solvent - dispersant combinations on yield stress.

\begin{tabular}{lc}
\hline Solvent - dispersant combination & $\begin{array}{c}\text { Yield stress } \\
\left(D / \mathrm{cm}^{2}\right)\end{array}$ \\
\hline EtOH-xylene-PE & $0 \cdot 07$ \\
EtOH-xylene-MFO & $0 \cdot 78$ \\
EtOH-xylene-T-X-100 & $0 \cdot 68$ \\
IPA-toluene-PE & $0 \cdot 13$ \\
IPA-toluene-MFO & $0 \cdot 78$ \\
IPA-toluene-T-X-100 & $0 \cdot 88$ \\
\hline
\end{tabular}

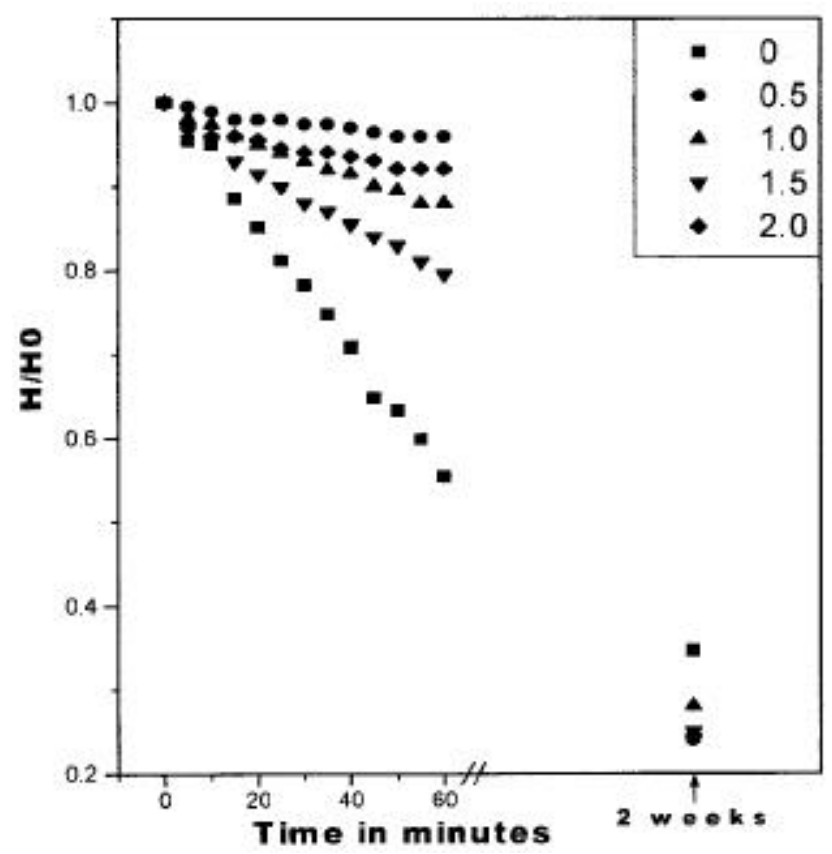

Figure 6. Sedimentation curves for $\mathrm{ZrO}_{2}$ in EtOH-xyl system with different concentrations of PE. 
Table 2. Effect of dispersant concentration on yield stress and sedimentation.

\begin{tabular}{llllll}
\hline PE $(\mathrm{wt} \%)$ & 0.0 & 0.5 & 1.0 & 1.5 & 2.0 \\
\hline Yield stress $\left(D / \mathrm{cm}^{2}\right)$ & 0.76 & 0.02 & 0.07 & 0.13 & 0.11 \\
Final $H / H 0$ & 0.347 & 0.24 & 0.28 & 0.25 & 0.245 \\
\hline
\end{tabular}

Table 3. Tape casting slurry composition.

\begin{tabular}{llr}
\hline Constituent & Role of constituent & Wt $\%$ \\
\hline $\mathrm{ZrO}_{2}$ & Ceramic powder & $57 \cdot 72$ \\
Phosphate ester & Dispersant & $0 \cdot 52$ \\
Xylene & Solvent & $16 \cdot 66$ \\
EtOH & Solvent & $4 \cdot 45$ \\
Cyclohexanone & Homogenizer & $0 \cdot 35$ \\
PEG 600 & Plasticizer & $2 \cdot 50$ \\
BBP & Plasticizer & $2 \cdot 50$ \\
PVB & Binder & $15 \cdot 30$ \\
\hline
\end{tabular}

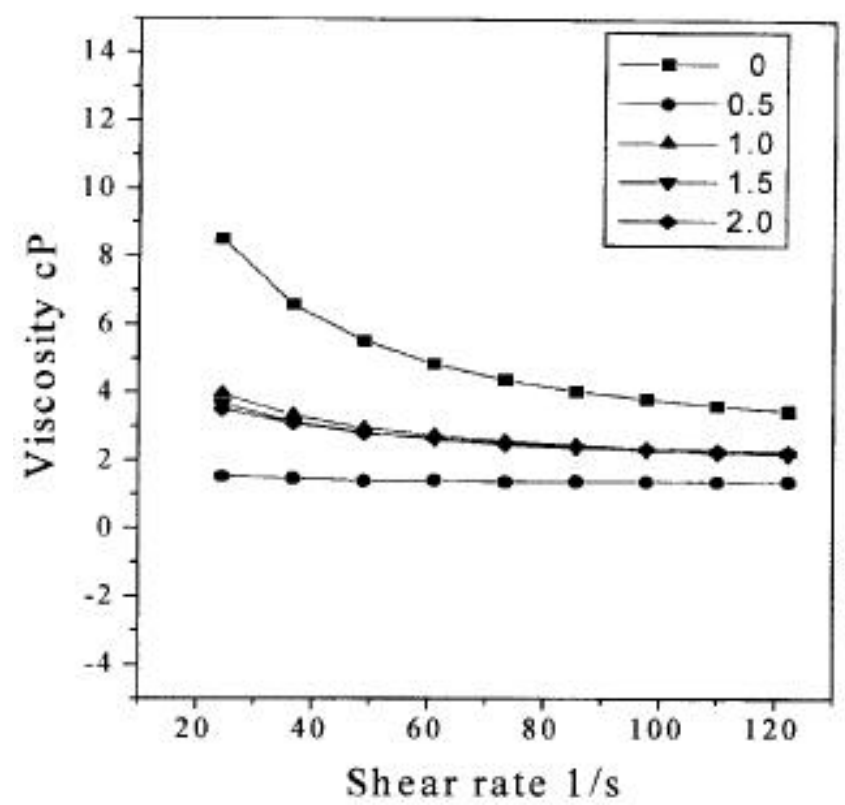

Figure 7. Rheological characteristics of $\mathrm{ZrO}_{2}$ in EtOH-xyl system with different concentrations of PE.

\subsection{Discussion}

Comparing the influence of different dispersants used for the given solvent system, it can be seen that irrespective of the solvent system, phosphate ester acts as the effective dispersant than MFO and T-X-100. As noted, dispersion can be because of electrostatic repulsion mechanism, steric hindrance mechanism or its combination. Menhaden fish oil contains polyunsaturated ester molecules, basically glyceryl esters of fatty acids such as oleate and linoleate (Mistler and Twiname 2000). This dispersant cannot be considered as a surfactant and steric stabilization is most likely to occur since no variation in

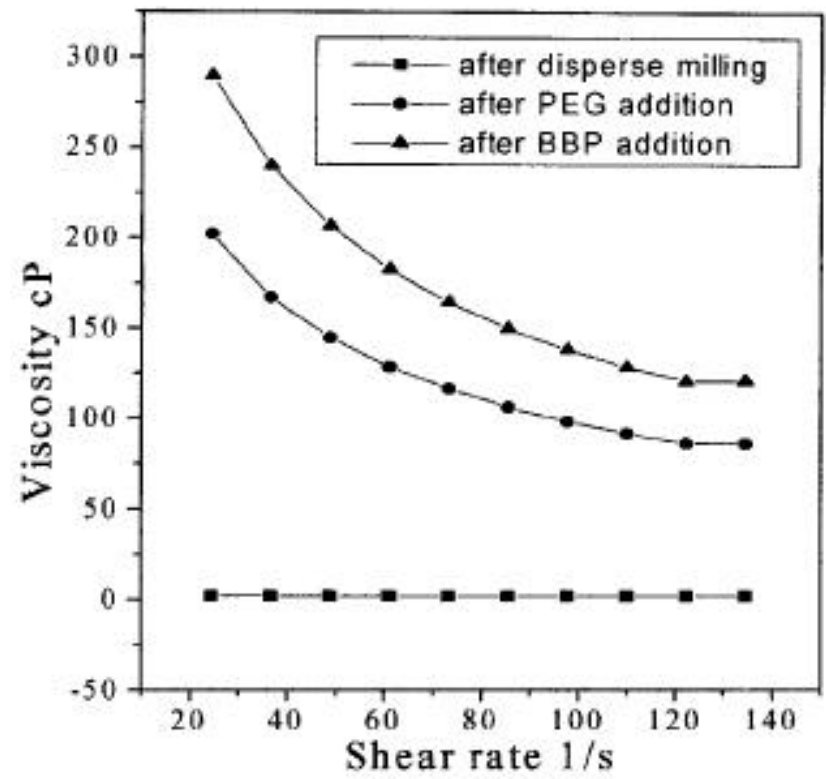

Figure 8. Rheological characteristics of $\mathrm{ZrO}_{2}$ slurry at different stages of slurry preparation.

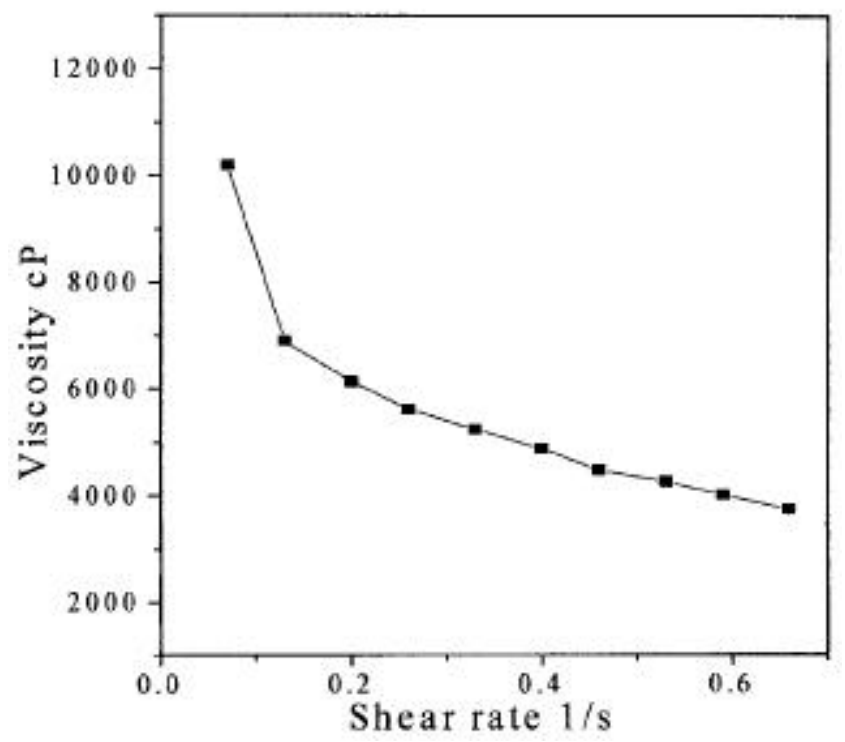

Figure 9. Rheological characteristics of $\mathrm{ZrO}_{2}$ tape casting slurry.

zeta potential occurs when this dispersant is added to slurry. Triton-X-100 also, because of its larger molecular size and longer chain, seem to act as a steric hindrant dispersant. On the other hand, in the case of phosphate 


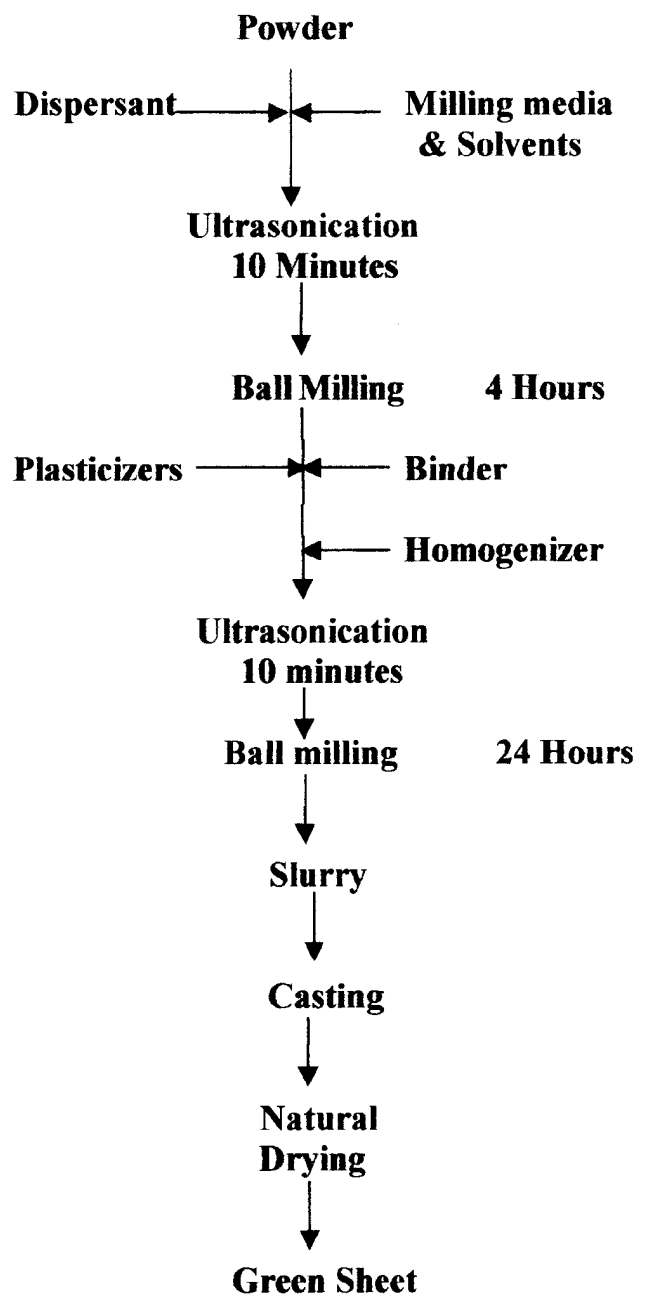

Figure 10. Flow chart for tape casting process.

ester, electrostatic repulsion mechanism is possible for oxides.

As per the manufacturer, the phosphate ester used is a combination of mono and dialkyl esters. The mono and dialkyl esters are highly acidic and exist as anions in aqueous/non-aqueous media. Monoalkyl esters can exist as dianions, monoanions or a combination of both depending on the dissociation constants of acidic esters. Acidity in both aqueous and non-aqueous systems has been confirmed by a measurable decrease in $\mathrm{pH}$ on addition of the phosphate ester, thus indicating some degree of dissociation and ionization of the phosphate ester even in the non-aqueous systems (Mistler and Twiname 2000; Bhaskar Reddy et al 2002). The free protons liberated during the dissociation of the phosphate ester are subsequently adsorbed on to the surface of the $\mathrm{ZrO}_{2}$, causing the metal oxide to take on a positively charged surface leading to electrostatic repulsive mecha- nism (Mistler and Twiname 2000; Bhaskar Reddy et al 2002).

Of the different solvent systems tried, 50:50 ratio of EtOH-xyl was found to be the best. This underlines the influence of right proportion of polarity (Feng and Dogan 2000) for the solvents.

\section{Conclusions}

The dispersion, viscosity and rheological characteristics of the tape casting slurry for PSZ were studied systematically at every stage of the slurry preparation. Based on these studies, the combination of $50: 50$ ratio of EtOHxyl solvent system and $0.5 \% \mathrm{PE}$ as the dispersant were used for achieving optimum dispersion. Using the optimized slurry with $58 \%$ of solid loading, which exhibits pseudoplastic characteristics, visibly defect free tapes of $\sim 50 \mu \mathrm{m}$ thickness and $55 \%$ green density were obtained.

\section{References}

Andreola F, Castellini E and Romagnoli M 2002 Am. Ceram. Soc. Bull. 8153

Annika Kristoffersson 1999 Water-based tape casting of ceramics and fabrication of ceramic laminates, $\mathrm{PhD}$ Thesis, Chalmers University of Technology, Sweden

Badini C, Fino P, Oetona A, Amelio C and Pasouale G 2001 Processing of multilayered SiC ceramics by tape casting and sintering (Rimini, Italy: Euromat)

Bhaskar Reddy S, Paramanand Singh P, Raghu N and Kumar V 2002 J. Mater. Sci. 37929

Bindu K and Raghu N 2004 J. Mater. Sci. (communicated)

Chartier T, Jorge E and Boch P 1993 J. Eur. Ceram. Soc. 11387

Deb S and Radhakrishna Bhat B V 2000 Bull. Mater. Sci. 23 109

Feng Jian-Huei and Dogan F 2000 J. Am. Ceram. Soc. 831681

Heuer A H and Hobbs V 1981 Advances in ceramics, Science and technology of zirconia (Westerville, USA: The American Ceram. Soc. Inc.) Vol. 3 p. 1

Jiyou Guo J and Lewis Jennifer A 2002 J. Am. Ceram. Soc. 83266

Kernan B D, Anqiang He and Heuer A H 2003 J. Am. Ceram. Soc. 86959

Mistler R E 1998 Am. Ceram. Soc. Bull. 7782

Mistler R E and Twiname E R 2000 Tape casting-Theory and practice (Westerville, USA: The American Ceramic Society) p. 23

Moreno R 1992a Am. Ceram. Soc. Bull. 101521

Moreno R 1992b Am. Ceram. Soc. Bull. 111647

Olieveria I R, Sepulveda P and Pandolfelli V C 2001 Ceram. Bull. 8047

Paik U, Hackley V A and Lee H W 1999 J. Am. Ceram. Soc. 82 833

Somia S, Yamamoto N and Yanagida H 1988 Advances in ceramics, Science and technology of zirconia 111 (Columbus, USA: The American Ceram. Soc. Inc.) Vol. A24, p. 3

Zhu X, Tang F, Suzuki T S and Sakka Y 2003 J. Am. Ceram. Soc. 86189 